\title{
Ein Nektarschluck im Flug
}

\author{
Petra Wester
}

\begin{abstract}
A long-standing paradigm in pollination ecology has been that New World hummingbirds hover and Old World birds perch when exploiting flowers for nectar. On the contrary, it is nowadays known that hummingbirds perch whenever possible and that nectarivorous Old World birds occasionally hover. However, no overview of hovering behaviour and its frequency in nectarivorous Old World birds exists. Here, observations of hovering behaviour in 81 species in 11 families of Old World birds are documented. Hovering behaviour occurs more frequently than thought before, not only in terms of the range of avian species but also in its occurrence within species. Neither the geographical distribution of bird species nor their traits such as size and body mass, are a limiting factor for hovering behaviour. In contrast, plant traits are important for the birds ' foraging behaviour. When perches at the plants are inadequate or lacking, hovering is more appropriate or necessary.
\end{abstract}

\section{Zusammenfassung}

Ein lange bestehendes Paradigma in der Bestäubungsbiologie war, dass neuweltliche Kolibris im Schwirrflug Nektar an Blüten trinken und altweltliche Vögel beim Blütenbesuch sitzen. Andererseits weiß man, dass Kolibris landen, wann immer möglich, und dass nektartrinkende Altweltvögel manchmal beim Blütenbesuch fliegen. Eine Übersicht über das Schwebverhalten und seine Häufigkeit bei nektartrinkenden Altweltvögeln gibt es jedoch nicht. Hier werden Beobachtungen über Schwebverhalten in 81 Arten aus 11 Familien von Altweltvögeln zusammengetragen. Schwebverhalten tritt häufiger auf als erwartet - in Bezug auf Vogelarten und Häufigkeit innerhalb der Arten. Begrenzende Faktoren für Schwebverhalten sind weder die geographische Verbreitung der Vogelarten, noch ihre Merkmale wie Größe und Gewicht, jedoch die Merkmale der Pflanzen. Bieten die Pflanzen keine oder nur unzureichende Sitzgelegenheiten, ist Schwebverhalten stärker angebracht oder sogar notwendig.

\section{Nektartrinkende Vögel und ihr Verhalten an den Blüten}

Nektartrinkende Vögel (Nektarivore), die Blüten bestäuben, gibt es in etwa 50 von 237 Vogelfamilien. Hochspezialisierte Nektarivore umfassen Kolibris in Amerika, Nektarvögel in Afrika, Asien und Australasien, Zuckervögel im südlichen Afrika, Honigfresser in Australasien sowie Mistelfresser und Papageien in Asien und Australasien. Opportunistische Nektartrinker wie Brillenvögel, Webervögel, Bülbüls oder Trupiale, die nur gelegentlich Nektar trinken, sind auch wichtige Bestäuber in der Alten und der Neuen Welt (WesTERKAMP 1999). Ein lange bestehendes Paradigma in der Bestäubungsbiologie war, dass nur Kolibris beim Nektartrinken an Blüten schweben (in der Luft stehen, ohne zu landen, Westerkamp 1990, Abb. 1). Da Kolibris auf die Neue Welt beschränkt sind, wurde angenommen, dass vogelbestäubte Blüten neuweltlicher Pflanzen von schwirrenden Kolibris und altweltliche vogelbestäubte Blüten nur von sitzenden Vögeln besucht werden (Abb. 2). Es wurde auch behauptet, dass vogelbestäubte neuweltliche Blüten meist Anpassungen an schwebende nektartrinkende Vögel aufweisen, wie die Ausrichtung zum freien Raum, wohingegen altweltliche Pflanzen den bestäubenden Vögeln Sitzgelegenheiten bieten. Allerdings gibt es auch neuweltliche Pflanzen mit Sitzgelegenheiten für Vögel und altweltliche Pflanzen, die diese nicht

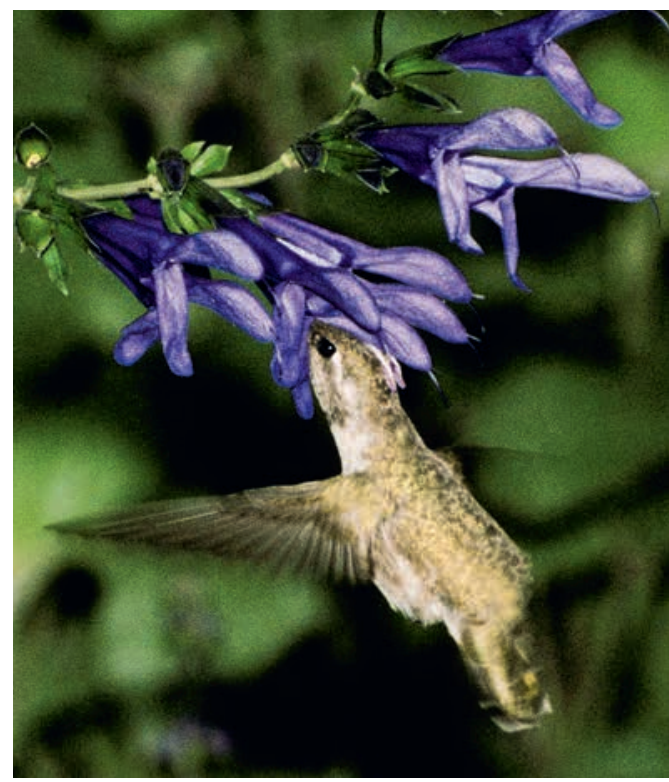

Abb. 1: Ein Kolibri, die Veilchenkopfelfe, im Schwirrflug an den Blüten des Guarani-Salbei in Kalifornien. (Foto: P. Wester) 
aufweisen. Außerdem landen Kolibris, wann immer möglich und viele neuweltliche nektartrinkende Vögel sitzen normalerweise beim Blütenbesuch. Darüber hinaus wurde festgestellt, dass nektartrinkende Altweltvogel bisweilen schweben (Abb. 3).

\section{Schwirrende Altwelt-Vögel}

Der folgende kurze Überblick über das Schwirrverhalten bei nektarivoren Altweltvögeln basiert auf Literaturdaten (meist kurze Beobachtungen) und eigenen Beobachtungen. Bei zumindest 81 Arten von Altweltvögeln wurde Schwebverhalten beim Blütenbesuch dokumentiert. Von den ungefähr 120 Nektarvogel-Arten gibt es mindestens bei 46 Arten (fast $40 \%$ ) Nachweise zum Schweben beim Nektartrinken. Dies wurde meist in Afrika beobachtet, aber auch in Südasien und Australasien. Dieses Verhalten wurde auch bei zehn anderen altweltlichen Vogelfamilien gefunden (Tab. 1, Wester 2014). Selbst bei uns in Europa, und sogar auch in Deutschland, im Mittelmeergebiet oder auch auf den Kanaren kann man Schwirrverhalten bei der Blaumeise (Cyanistes caeruleus), der Mönchsgrasmücke (Sylvia atricapilla), dem Fitis (Phylloscopus trochilus), dem Zilpzalp (Phylloscopus collybita) und dem Kanaren-Zilpzalp (Phylloscopus canariensis) beobachten. Im Flug besucht werden u.a. Blüten von Obstpflanzen (Prunus domestica, Rosaceae; Musa sp., Musaceae), und Zierpflanzen (z. B. Erythrina caffra, Fabaceae; Grevillea robusta, Proteaceae; Eucalyptus spp., Myrtaceae; Aloe vera, Kniphofia sp., Asphodelaceae; Melianthus minor, Melianthaceae). Besucht werden aber auch einheimische Arten (z. B. Anagyris foetida, Fabaceae; Scrophularia calliantha, S. sambucifolia, Isoplexis canariensis, Scrophulariaceae; Acer sp., Sapindaceae; Salix sp., Salicaceae, Westerkamp 1996, 1999, Wester 2014).

\section{Verschiedene Schwebflug-Verhaltensweisen}

Der schnelle Schwirrflug der Kolibris ist sehr präzise. In Kalifornien konnte die Autorin eine Veilchenkopfelfe (Calypte costae) dabei beobachten, als sie sich beim Besuch von Blüten des GuaraniSalbei (Salvia guaranitica, Lamiaceae) $360^{\circ} \mathrm{um}$ ihre Längsachse drehte (Abb. 1). Der Schwirrflug der Kolibris unterscheidet sich vom Trillerflug (Flattern) anderer Vögel wie Nektarvögel, die

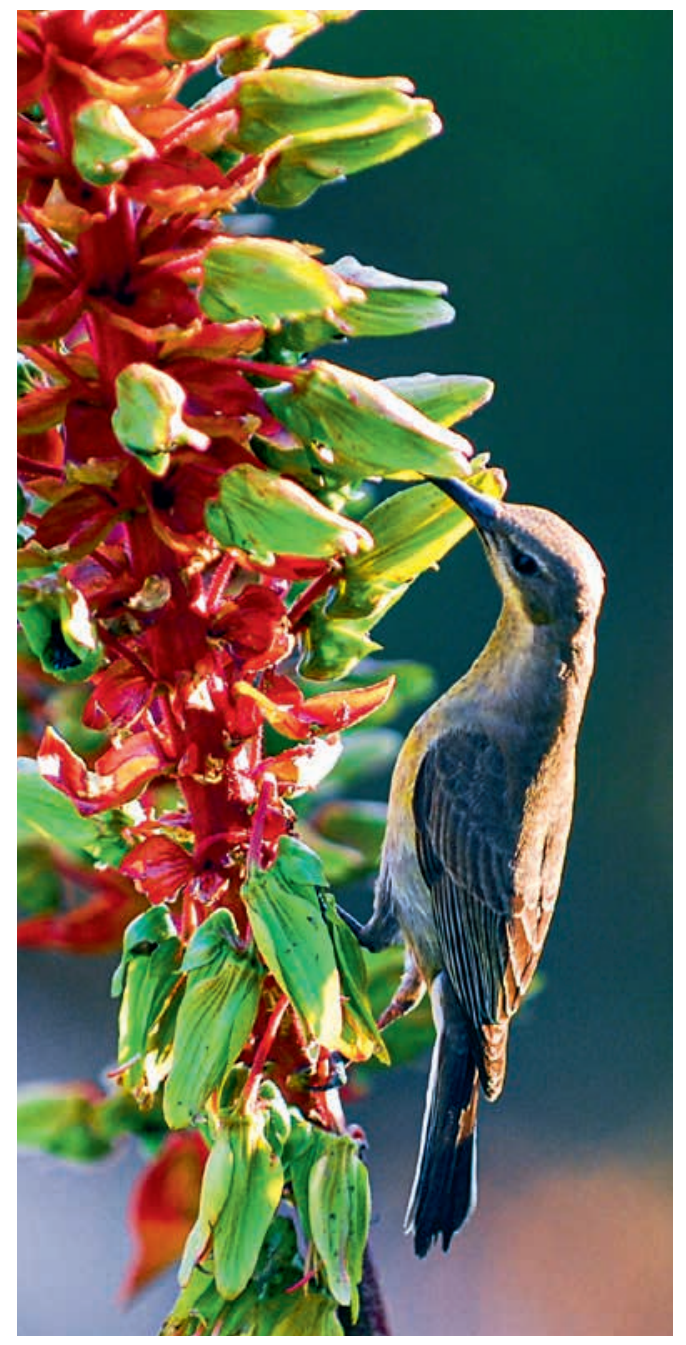

Abb. 2: Blütenbesuchender Malachit-Nektarvogel sitzend auf Melianthus major (Melianthaceae). (Foto: P. Wester)

unterschiedliche Funktionsmorphologie der Vögel widerspiegelnd. Dank ihrer unterschiedlichen Flügelbewegung und -Frequenz können Kolibris länger schwirren (in einem Experiment für länger als 50 Minuten) als andere Vogelarten. Eine frühe Untersuchung des Schwebflugs von Nektarvögeln bezeichnete diesen als „ungeschicktes Manöver“. Hingegen hat die Autorin den Eindruck, dass der Nektarvogel auch akrobatisch sein kann, vor allem der Malachit-Nektarvogel (Nectarinia famosa, Abb. 3). Schwebende Nektarvögel sind auch in der Lage, rückwärts zu fliegen. Sie können ihre Schnäbel exakt in Blüten einführen, ohne diese 


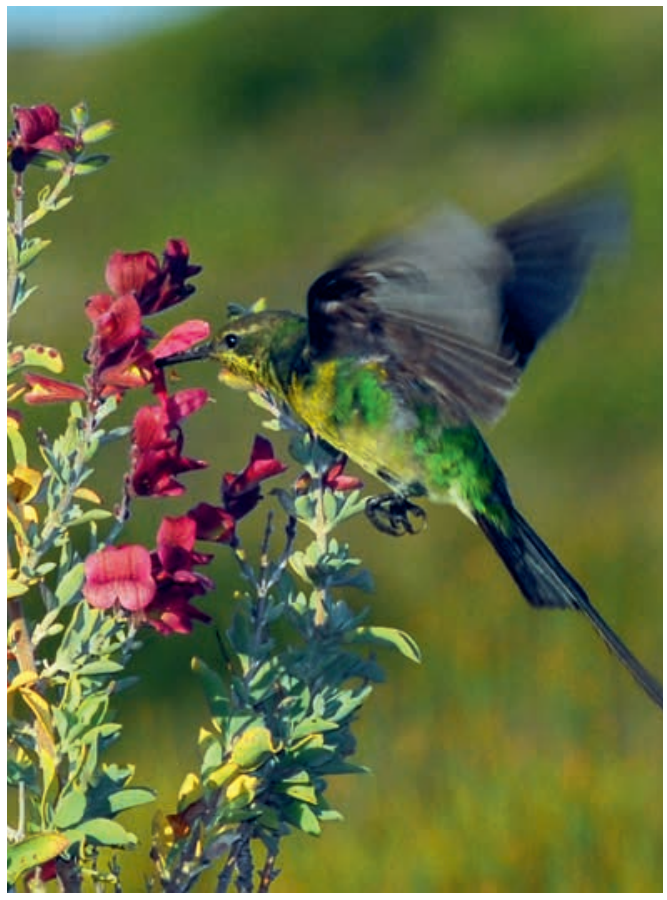

Abb. 3: Fliegender Malachit-Nektarvogel besucht Blüten des Lanzett-Salbei (Salvia lanceolata, Lamiaceae) in Südafrika. (Foto: P. WESTER) zu beschädigen. Neben dem Schwebflug (Flug auf der Stelle) gibt es den Rüttelklammerflug (sitzen oder festklammern und gleichzeitig mit den Flügeln schlagen, Abb. 4). Ihn kann man seltener beobachten bei Nektarvögeln, Laubsängern, Kolibris und einigen nektartrinkenden Fledermäusen.

\section{Häufigkeit des Schwebflug-Verhaltens}

Die Häufigkeit des Schwebflugs bei Altweltvögeln reicht von nie bis ausschließlich (Tab. 1). Die Bedeutung des Schwebflugs für den Rotnacken-Honigfresser (Acanthorhynchus tenuirostris, Meliphagidae) spiegelt sich in dessen Trivialnamen wider; man nennt ihn den „Kolibri Australiens“. Vergleicht man die verschiedenen Vögel, die bei der Nahrungsaufnahme schweben, fällt auf, dass ihre geographische Verbreitung, systematische $\mathrm{Zu}$ gehörigkeit und morphologischen Merkmale (z. B. Größe oder Körpergewicht) nicht der limitierende Faktor für Schwirrverhalten sind. Beispielsweise hat die Autorin den relativ schweren MalachitNektarvogel (ca. 15-20 g) häufiger im Schwebflug an südafrikanischen Salbei-Arten (Salvia spp., Abb. 3) beobachtet als den kleineren und leichte-

Tabelle 1: Taxa von Altwelt-Vögeln, die beim Schwirrflug an Blüten beobachtet wurden *)

\begin{tabular}{|l|l|l|}
\hline $\begin{array}{l}\text { Vogelfamilien } \\
\text { (Artenzahl mit Schwirrflug/ } \\
\text { Gesamt-Artenzahl in Alter Welt) }\end{array}$ & $\begin{array}{l}\text { Häufigkeit des } \\
\text { Schwirrflugs }\end{array}$ & Region \\
\hline Sectariniidae: Nektarvögel (46/126) \& & selten bis ausschließlich & $\begin{array}{l}\text { meist Afrika, auch SO-Asien u. } \\
\text { Australien } \\
\text { S(O)-Asien }\end{array}$ \\
\hline Dicaeidae: Mistelfresser (2/48) & oft bis ausschließlich & Philippinen \\
\hline Promeropidae: Zuckervögel (1/2) & oft & Südafrika \\
\hline Eurylaimidae: Nektarjalas (1/20) & manchmal & Madagaskar \\
\hline Meliphagidae: Honigfresser (15/183) & selten bis ausschließlich & Australien, Südwestpazifische Inseln \\
\hline Zosteropidae: Brillenvögel (3/125) & manchmal & Südafrika, Réunion \\
\hline Cisticolidae: Prinien (1/159) & selten & Südafrika \\
\hline Pycnonotidae: Bülbüls (1/151) & selten & Südafrika \\
\hline Phylloscopidae: Laubsänger (4/77) & selten bis häufig & Europa, Kanaren \\
\hline Sylviidae: Grasmücken \& Myzornis (2/69) & & Europa, Südasien \\
\hline Paridae: Meisen (1/48) & & Europa \\
\hline
\end{tabular}

*) für Details siehe Wester (2014) 


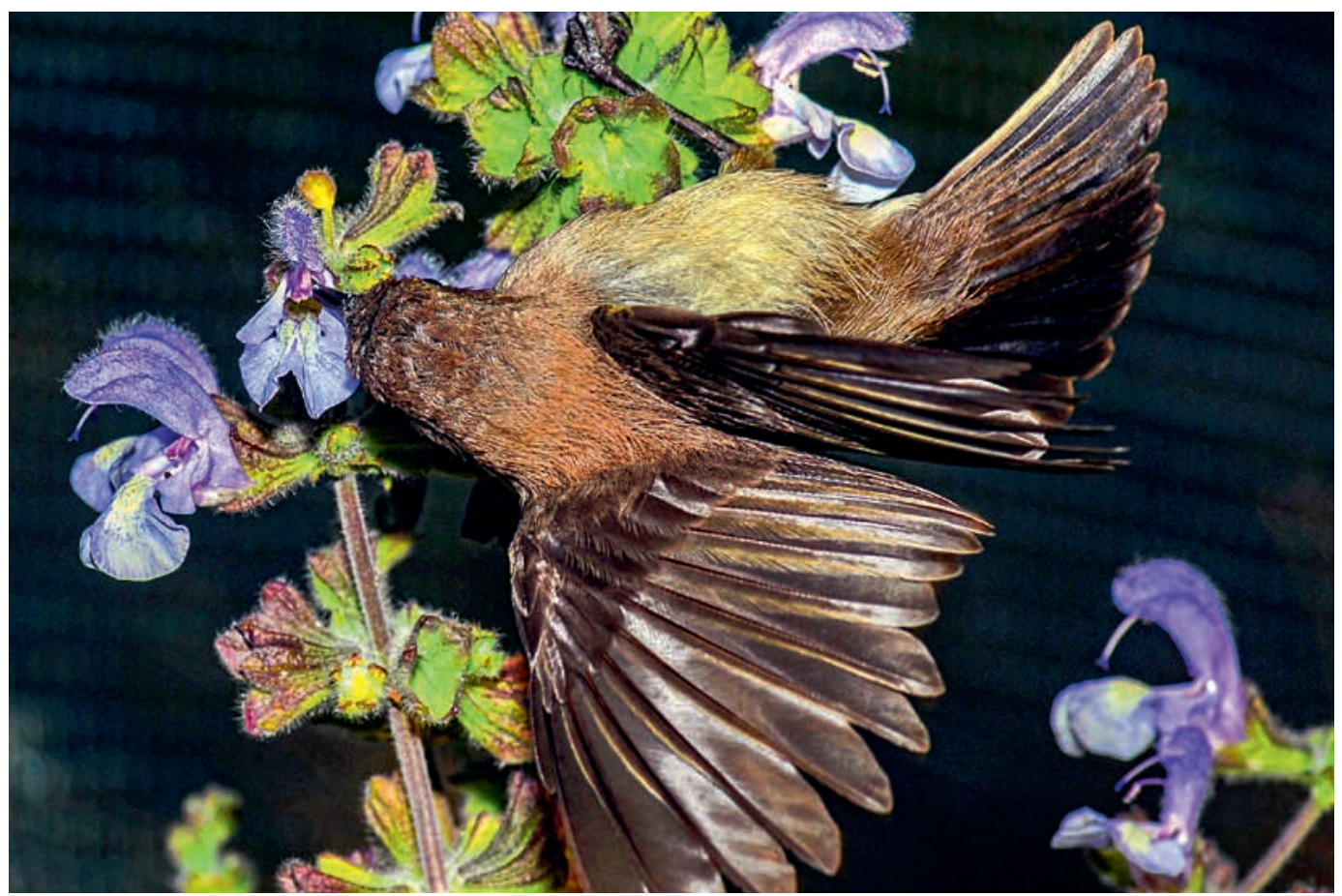

Abb. 4: Weiblicher Blauband-Nektarvogel besucht eine bienenbestäubte Blüte von Salvia africana-caerulea im Rüttelklammerflug. (Foto: P. Wester)

ren Blauband-Nektarvogel (Cinnyris chalybeus, ca. 7-9 g, Abb. 4, 5, Wester 2013). Sogar der größte und schwerste Nektarvogel, der Riesen-Nektarvogel (Dreptes thomensis, ca. 18-28 g) und der viel schwerere Kap-Honigvogel (Promerops cafer, ca. $32-37 \mathrm{~g}$ ) sind in der Lage, Blüten im Schwebflug zu besuchen.

\section{Die Bedeutung der Pflanzenmerkmale}

Pflanzenmerkmale scheinen die entscheidenden Faktoren zu sein, die bestimmen, ob Vögel beim Blütenbesuch schweben oder sitzen. Blüten (oder Teile) von Pflanzen, die stabile Sitzgelegenheiten bieten, können bevorzugt von Vögeln besucht werden, die beim Blütenbesuch sitzen. Wenn Sitzgelegenheiten bei Pflanzen jedoch unzulänglich sind oder sogar fehlen, ist der Schwebflug eher angebracht oder notwendig. Angepasst an (schwirrende) Kolibris, haben viele amerikanische Pflanzen zum freien Raum hin orientierte Blüten, aber keine stabilen Sitzgelegenheiten. Der auch in der Alten Welt eingebürgerte andine Baum-Tabak (Nicotiana glauca, Solanaceae)

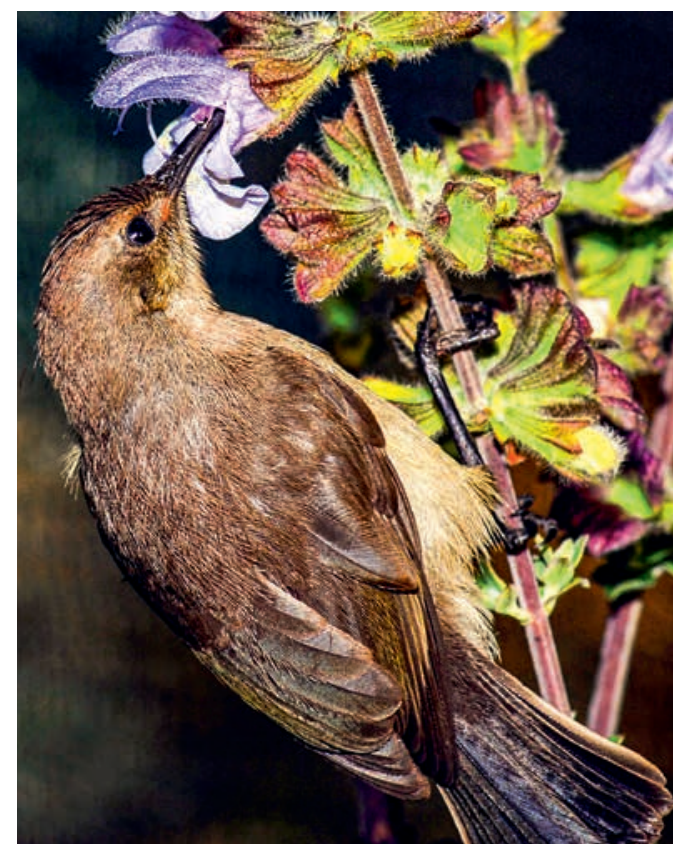

Abb. 5: Derselbe Blauband-Nektarvogel besucht die benachbarte Blüte von Salvia africana-caerulea im Sitzen. (Foto: P. Wester) 
wird in Südafrika von Malachit-Nektarvögeln aufgesucht, die die Blüten meist im Schwebflug besuchen (Geerts \& PAuw 2009). Auch in der Alten Welt gibt es Pflanzenarten, die keine für Vögel adäquaten Sitzgelegenheiten haben, zum Beispiel das westafrikanische Springkraut (Impatiens sakeriana, Balsaminaceae). Die Blüten dieser Art stehen an langen Blütenstielen, die zum freien Raum hin orientiert sind und die Nektarvögel meistens zwingen, sie im Schwebflug zu besuchen (JANEČEK et al. 2011). Ähnliche Beispiele sind das Ostafrikanische Glockenblumengewächs Canarina eminii (Campanulaceae), welches von schwebenden Nektarvögeln besucht wird, und die Schamblume (Aeschynanthus pulcher, Gesneriaceae), deren Blüten in Indonesien von Spinnenjägern im Schwebflug besucht werden.

Es ist sehr wahrscheinlich, dass Schwebverhalten beim Blütenbesuch bei weiteren altweltlichen Vogelarten vorkommt und dass Pflanzen, die an diese Vögel angepasst sind, sogar übersehen wurden.

\section{Literatur}

Geerts, S. \& Pauw, A. 2009: African sunbirds hover to pollinate an invasive hummingbird-pollinated plant. - Oikos 118: 573-579.

Janeček, Š., Patáčivá, E., Bartoš, M., Padyšáková, E. Spitzer, L. \& R. Tropek 2011: Hovering sunbirds in the Old World: occasional behaviour or evolutionary trend? - Oikos 120: $178-183$.

Wester, P. 2013. Sunbirds hover at flowers of Salvia and Lycium. - Ostrich 84: 27-32.

Wester, P. 2014. Feeding on the wing: hovering in nectardrinking Old World birds - more common than expected. Emu 114: 171-183.

Westerkamp, C. 1990. Bird-flowers: hovering versus perching exploitation. - Bot. Acta 103: 366-371.

Westerkamp, C. 1996. Heimische Blumenvögel. - Palmengarten 60: 17-24.

Westerkamp, C. 1999. Vogelblumen - Blumenvögel. - In: Zizka, G. \& Schneckenburger, S. (Hrsg.): Blütenökologie. pp. 88-94. - Frankfurt.

\section{Anschrift der Autorin}

Dr. Petra Wester, School of Life Sciences, University of KwaZulu-Natal, P.B. X01, Pietermaritzburg 3209, Südafrika, E-Mail: westerpetra3@gmail.com http://orchid.org/0000-0002-6514-8159 